\title{
The Linear Two-Point Boundary-Value Problem on an Infinite Interval
}

\author{
By T. N. Robertson
}

\begin{abstract}
A numerical method, using finite-difference approximations to the second-order differential equation, is given which tests the suitability of the finite point chosen to represent infinity before computing the numerical solution. The theory is illustrated with examples and suggestions for further applications of the method are presented.
\end{abstract}

1. Introduction. Consider the linear two-point boundary-value problem on an infinite interval

$$
L y=-y^{\prime \prime}+p(x) y^{\prime}+q(x) y=f(x) \text { with } y(a)=\alpha, y(\infty)=0 .
$$

More general boundary conditions will be considered later.

A typical method is to apply the second boundary condition at a finite point or at several finite points and observe the variation of the solutions (Fox [2]). The basic objective is to obtain an accurate numerical solution in a relatively small finite interval starting at $a$. The proposed method, involving finite-difference approximations, finds a solution over such an interval and also produces values $b$ and $\beta$ such that the solution of $(1)$ on $[a, b]$ is given by the solution of

$$
L y=f \quad \text { with } y(a)=\alpha, y(b)=\beta .
$$

Thus a finite two-point boundary-value problem is derived for which solutions can be sought by application of any appropriate method.

The procedure here proposed is to examine $y^{(N)}(x)$, the solution of

$$
L y=f \quad \text { with } y(a)=\alpha, y\left(b^{(N)}\right)=0
$$

under conditions which ensure that $y^{(N)}(x) \rightarrow y(x)$ where $y(x)$ satisfies (1).

2. Preliminaries. The existence and uniqueness of the solution of the two-point boundary-value problem for $L y=f$ on a finite interval is guaranteed if $p, q$ and $f$ are continuous with $q>0$ (Keller [3]). Thus these conditions are sufficient for $y^{(N)}(x)$ to exist for any sequence of points $\left\{b^{(N)}\right\}$.

The asymptotic behavior of solutions of second-order differential equations is discussed in Bellman [1]. In particular, the two independent solutions of an equation of the form

$$
\begin{aligned}
& y^{\prime \prime}-(1+\phi(x)) y=0 \\
& \text { with } \phi(x) \rightarrow 0 \text { as } x \rightarrow \infty \text { and } \\
& \int^{\infty}|\phi(t)|^{n} d t<\infty, \text { for some } n,
\end{aligned}
$$

Received November 10, 1970.

AMS 1969 subject classifications. Primary 6562.

Key words and phrases. Two-point boundary-value problem, second-order linear differential equation, finite-difference approximation, condition at infinity.

Copyright @ 1971, American Mathematical Society 
have asymptotic expansions which are exponential functions, one with a positive and one with a negative exponent. Since $L y=-y^{\prime \prime}+p y^{\prime}+q y=0$ can be transformed into (4) under very broad conditions, we can state for our problem that if

$$
0<Q_{*}<q(x)<Q^{*} \text { and }|p(x)|<P^{*}, \quad|f(x)|<F^{*} \text { on }[a, \infty)
$$

then the general solution of $L y=f$ can be expressed as

$$
y(x)=A y_{1}(x)+B y_{2}(x)+Y(x)
$$

where $y_{1}(x) \rightarrow 0$ and $y_{2}(x) \rightarrow \infty$ as $x \rightarrow \infty$ and $Y(x)$ is a particular solution.

Thus for $y^{(N)}(x)$ satisfying (3) we can write

$$
y^{(N)}(x)=A^{(N)} y_{1}(x)+B^{(N)} y_{2}(x)+Y(x)
$$

where we require that $Y(x) / y_{2}(x) \rightarrow 0$ as $x \rightarrow \infty$. Now applying the boundary conditions

$$
\begin{aligned}
& \alpha=y^{(N)}(a)=A^{(N)} y_{1}(a)+B^{(N)} y_{2}(a)+Y(a), \\
& 0=y^{(N)}\left(b^{(N)}\right)=A^{(N)} y_{1}\left(b^{(N)}\right)+B^{(N)} y_{2}\left(b^{(N)}\right)+Y\left(b^{(N)}\right),
\end{aligned}
$$

we have

$$
\begin{aligned}
& A^{(N)}=\frac{(\alpha-Y(a)) y_{2}\left(b^{(N)}\right)+Y\left(b^{(N)}\right) y_{2}(a)}{y_{1}(a) y_{2}\left(b^{(N)}\right)-y_{1}\left(b^{(N)}\right) y_{2}(a)}, \\
& B^{(N)}=-\frac{(\alpha-Y(a)) y_{1}\left(b^{(N)}\right)+Y\left(b^{(N)}\right) y_{1}(a)}{y_{1}(a) y_{2}\left(b^{(N)}\right)-y_{1}\left(b^{(N)}\right) y_{2}(a)},
\end{aligned}
$$

so that $A^{(N)} \rightarrow(\alpha-Y(a)) / y_{1}(a)$ and $B^{(N)} \rightarrow 0$ as $N \rightarrow \infty$, and

$$
y(x)=\lim _{N \rightarrow \infty} y^{(N)}(x)=\frac{\alpha-Y(a)}{y_{1}(a)} y_{1}(x)+Y(x)
$$

satisfies $L y=f$ and $y(a)=\alpha, y(\infty)=0$.

3. Numerical Method. For some initial choice of $h$ and $N$ define a uniform net

$$
x_{i}=a+j h, \text { for } j=0,1,2, \cdots, N,
$$

and let $\bar{u}^{(N)}$ be the solution of the finite-difference approximation, for which a typical choice is

$$
\begin{aligned}
L_{h} u_{j}^{(N)} & =-\frac{u_{j+1}^{(N)}-2 u_{j}^{(N)}+u_{j-1}^{(N)}}{h^{2}}+p\left(x_{j}\right) \frac{u_{i+1}^{(N)}-u_{j-1}^{(N)}}{2 h}+q\left(x_{i}\right) u_{j}^{(N)} \\
& =f\left(x_{i}\right) \text { for } j=1,2, \cdots, N,
\end{aligned}
$$

with $u_{0}^{(N)}=\alpha$ and $u_{N+1}^{(N)}=0$.

Under the conditions (5) and assuming that $p, q, f$ and thus $y^{(N)}(x)$ are sufficiently smooth, the stability of the linear difference operator is assured (Keller [3]) and the difference equations have a unique solution with

$$
\left|u_{i}^{(N)}-y^{(N)}\left(x_{i}\right)\right|=O\left(h^{2}\right) .
$$


We write the system of equations (6) in the form

$$
A^{(N)} \bar{u}^{(N)}=\bar{d}^{(N)}
$$

where

$$
A^{(N)}=\left(\begin{array}{ccccc}
b_{1} & & \multicolumn{2}{c}{c_{1}} & \\
a_{2} & b_{2} & & c_{2} & \\
\ddots & \ddots & \ddots & \ddots \\
& \ddots & \ddots & \\
& a_{N-1} & b_{N-1} & c_{N-1} \\
& & a_{N} & b_{N}
\end{array}\right), \quad \bar{u}^{(N)}=\left(\begin{array}{c}
u_{1} \\
u_{2} \\
\vdots \\
u_{N}
\end{array}\right), \quad \bar{d}^{(N)}=\left(\begin{array}{c}
d_{1} \\
d_{2} \\
\vdots \\
d_{N}
\end{array}\right)
$$

with

$$
\begin{array}{ll}
a_{i}=-\frac{1}{2}\left(1+\frac{h}{2} p\left(x_{j}\right)\right), & \text { for } j=2,3, \cdots, N, \\
b_{i}=1+\frac{h^{2}}{2} q\left(x_{i}\right), & \text { for } j=1,2,3, \cdots, N, \\
c_{j}=-\frac{1}{2}\left(1-\frac{h}{2} p\left(x_{i}\right)\right), & \text { for } j=1,2, \cdots, N-1, \\
d_{1}=\frac{h^{2}}{2} f\left(x_{1}\right)-a_{1} \alpha \text { and } d_{i}=\frac{h^{2}}{2} f\left(x_{j}\right), & \text { for } j=2,3, \cdots, N .
\end{array}
$$

Now replace (7) by the equivalent system

$$
P^{(N)} \bar{u}^{(N)}=\bar{e}^{(N)}
$$

where

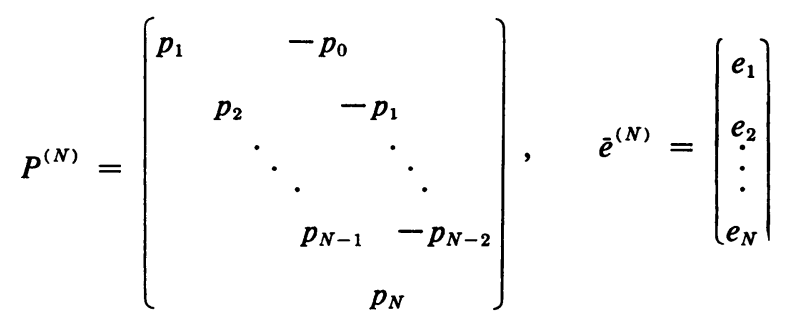

with

$$
\begin{array}{cc}
c_{i} p_{i}+b_{i} p_{i-1}+a_{i} p_{i-2}=0, & \text { for } j=1,2, \cdots, N, \\
c_{i} e_{i}-a_{j} e_{i-1}=-d_{i} p_{i-1}, & \text { for } j=1,2, \cdots, N,
\end{array}
$$

defining $p_{-1}=0, p_{0}=1, e_{0}=\alpha$ and $c_{N}=a_{N}$.

Here we are following the algorithm proposed by Olver [4] for the solution of second-order difference equations. Evidently $\bar{u}^{(N)}$ is readily obtained by back substitution from (8) but a more important point is that we can test the suitability of $N$ without computing $\bar{u}^{(N)}$. 
For, suppose $N$ is replaced by $N+1$ so now

$$
P^{(N+1)} \bar{u}^{(N+1)}=\bar{e}^{(N+1)} \text { where } u_{0}^{(N+1)}=\alpha, u_{N+2}^{(N+1)}=0 .
$$

Hence $p_{k} u_{k}^{(N+1)}-p_{k-1} u_{k+1}^{(N+1)}=e_{k}, k=1,2, \cdots, N+1$, and, from (8), $p_{k} u_{k}^{(N)}-$ $p_{k-1} u_{k+1}^{(N)}=e_{k}, k=1,2, \cdots, N$. Therefore

$$
\begin{aligned}
u_{k}^{(N+1)}-u_{k}^{(N)} & =\frac{p_{k-1}}{p_{k}}\left(u_{k+1}^{(N+1)}-u_{k+1}^{(N)}\right) \\
& =\frac{p_{k-1}}{p_{N}}\left(u_{N+1}^{(N+1)}-u_{N+1}^{(N)}\right)
\end{aligned}
$$

and since $u_{N+1}^{(N)}=0$ we have that

$$
u_{k}^{(N+1)}-u_{k}^{(N)}=\frac{p_{k-1}}{p_{N}} \frac{e_{N+1}}{p_{N+1}}, \text { for } k=1,2, \cdots, N+1 .
$$

Thus to apply the criterion

$$
\left\|\bar{u}^{(N)}-\bar{u}^{(N+1)}\right\|<\epsilon
$$

a first test is simply to examine $u_{N+1}^{(N+1)}=e_{N+1} / p_{N+1}$. If this is not small enough, set $N$ to $N+1$ and continue. Then, when this is met also require that

$$
\max _{1 \leqq k \leqq N}\left|\frac{p_{k-1} e_{N+1}}{p_{N} p_{N+1}}\right|<\epsilon .
$$

For the starting value of $h$, therefore, we are able to test that our finite replacement for infinity is reasonable before computing the solution of the finite-difference approximation.

Now replace $h$ by $h / 2$ and apply a criterion based on difference corrections to terminate the process. In our case, an $O\left(h^{4}\right)$ approximation is

$$
\tilde{u}_{i} \equiv u_{j}(h / 2)+\frac{1}{3}\left(u_{j}(h / 2)-u_{j}(h)\right)
$$

at points common to the mesh size $h$ and $h / 2$ and we have a theoretically justified test of accuracy using repeated difference corrections (Pereyra [5]).

Moreover, at any point $x_{i}$ for which

$$
\frac{1}{3}\left|u_{j}(h / 2)-u_{j}(h)\right|<\epsilon
$$

we can take $b=x_{i}$ and $\beta=u_{i}(h / 2)$ in the derived finite two-point boundary-value problem (2).

It will often be computationally difficult to refine the mesh size so that (10) is satisfied at all points. But over the finite interval accurate solutions can be sought using any appropriate method (including initial-value methods-shooting, backwards shooting-of high order) and refinement of the mesh size to give accurate $h \rightarrow 0$ extrapolation can be achieved.

4. Numerical Results. A FORTRAN program for an IBM 1620 was prepared and used to test the method. Two examples are presented:

(1) $L y=-y^{\prime \prime}-2 y^{\prime}+2 y=e^{-2 x}$ with $y(0)=1, y(\infty)=0$ for which the exact solution $y_{e}(x)=\frac{1}{2} e^{-(1+\sqrt{ } 3) x}+\frac{1}{2} e^{-2 x}$ tends rapidly to zero.

$$
\begin{array}{llllll}
\text { With } \epsilon=10^{-8} & \text { and } & h=1 & 0.5 & 0.25 \\
& \text { then } & N=9 & 18 & 34 \\
& \text { so that } & x= & 10 & 9.5 & 8.75
\end{array}
$$


were the values produced to represent infinity.

These results are entirely reasonable since $y_{e}(x)<10^{-8}$ for $x>9$.

(2) Fox [2 p. 91], gives the example $L y=-y^{\prime \prime}+(1+1 / x) y=1 / x^{2}$ with $y(1)=$ $y(\infty)=0$ for which the solution tends to zero relatively slowly. The equation $L y=0$ can be transformed into the confluent hypergeometric equation.

With $\epsilon=10^{-4}$, Fig. 1 shows the numerical results on the interval $[1,10]$ showing the effect of mesh size.

$$
\begin{array}{rlllll}
\text { For } & h= & 2 & 1 & 0.5 & 0.25 \\
& N=46 & 78 & 124 & 187 \\
\text { so that } & x= & 94 & 78 & 62.5 & 47
\end{array}
$$

are the values at which the second boundary condition is applied. In particular, at $x=9$,

$$
\begin{array}{ccccc}
h & 1 & 0.5 & 0.25 \\
y(9) & 0.011961 & 0.011958 & 0.011957 \\
\Delta y \times 10^{6} & & 3 & & 1
\end{array}
$$

which illustrates (10) so that the derived problem could be taken as

$$
L y=\frac{1}{x^{2}} \quad \text { with } \quad y(1)=0, \quad y(9)=0.011957
$$

Numerical results for this finite two-point problem were obtained using an iterative difference scheme. These confirmed the values shown in Fig. 1.

5. Further Applications.

(a) More General Boundary Conditions. Consider

$$
\begin{aligned}
L y=f \quad \text { with } \quad \alpha_{1} y(a)-\beta_{1} y^{\prime}(a) & =\gamma_{1}, \\
\alpha_{2} y(\infty)+\beta_{2} y^{\prime}(\infty) & =\gamma_{2},
\end{aligned}
$$

for $\alpha_{i}, \beta_{i} \geqq 0, i=1,2$, and $\alpha_{2} \neq 0$.

Replacing $y$ by $y+\gamma_{2} / \alpha_{2}$ gives boundary conditions in the form

$$
\begin{aligned}
\alpha_{1} y(a)-\beta_{1} y^{\prime}(a) & =\alpha, \\
\alpha_{2} y(\infty)+\beta_{2} y^{\prime}(\infty) & =0 .
\end{aligned}
$$

Now, apart from additional complication, the verification that $y^{(N)}(x) \rightarrow y(x)$ goes through as before.

Similarly, with the new more appropriate finite-difference approximation

$$
\begin{gathered}
L_{h} u_{i}=f\left(x_{i}\right), \quad \text { for } j=0,1,2, \cdots, N+1, \text { and } \\
\alpha_{1} u_{0}-\beta_{1} \frac{u_{1}-u_{-1}}{2 h}=\alpha, \\
\alpha_{2} u_{N+1}-\beta_{2} \frac{u_{N+2}-u_{N}}{2 h}=0,
\end{gathered}
$$

we have a tridiagonal system of $N+2$ equations for $u_{0}, u_{1}, \cdots, u_{N+1}$ which is reducible as before. 
480

T. N. ROBERTSON

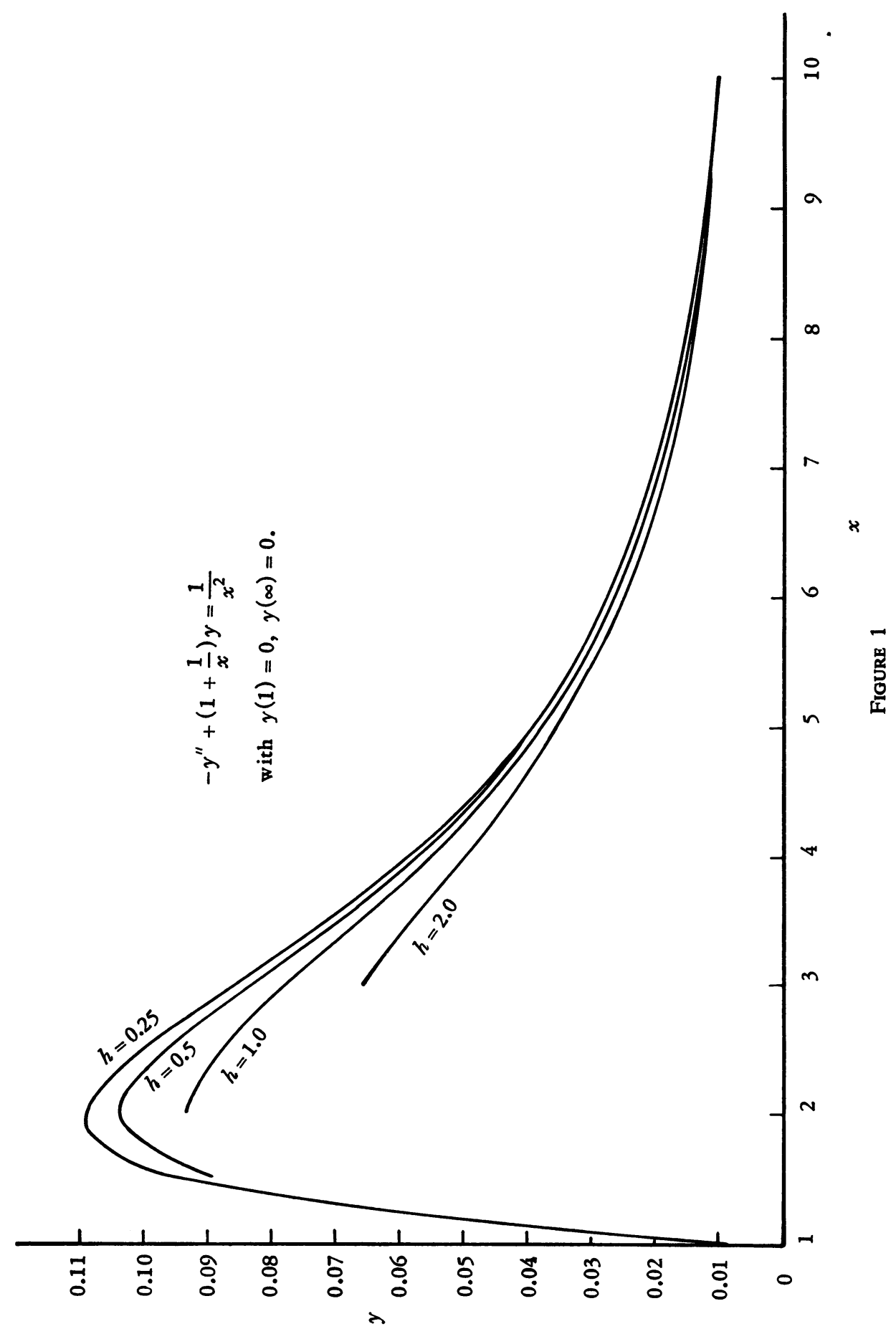


(b) Systems of Second-Order Linear Equations. Finite-difference methods applied to such a system produce, corresponding to the tridiagonal matrix $A$ in (7), a block tridiagonal corresponding to the matrix $P$ in (8). Thus the suitability of the point at which the second boundary condition is applied can again be tested before computing the solution to the finite-difference approximation.

(c) Second-Order Elliptic Partial Differential Equations.

(i) On an unbounded region. As a guide for the choice of the finite position of outer boundary (often taken to be a circular arc) we can set one of the spatial variables equal to a constant and examine the resultant ordinary differential equation by the present method.

(ii) On a semi-infinite region. Using five-point approximations in a rectangular spatial mesh over the region

$$
R:|x| \leqq K
$$

the finite-difference approximation can be expressed in terms of a block tridiagonal matrix (Varga [6])

$$
A \bar{u}=\bar{k}
$$

where

$$
A=\left(\begin{array}{cccc}
B_{1} & \multicolumn{2}{c}{C_{1}} & \\
A_{2} & B_{2} & & C_{2} \\
\ddots & \ddots & \ddots & \ddots \\
& A_{N-1} & B_{N-1} & C_{N-1} \\
& & A_{N} & B_{N}
\end{array}\right) .
$$

Here the square submatrices are of order $n$ where $n$ is the number of points along the horizontal mesh lines and $N$ is the number of horizontal lines in $R$. Thus the reduction to a block two band matrix will again permit a test of $N$.

Mathematics Department

Occidental College

Los Angeles, California 90041

1. R. Bellman, Stability Theory of Differential Equations, McGraw-Hill, New York, 1953. MR 15, 794.

2. L. Fox, Numerical Solution of Two-Point Boundary Value Problems in Ordinary Differential Equations, Clarendon Press, Oxford, 1957.

3. H. B. Keller, Numerical Methods for Two-Point Boundary-Value Problems, GinnBlaisdell, Waltham, Mass., 1968. MR 37 \#6038.

4. F. W. J. OlVER, "Numerical solution of second-order linear difference equations," J. Res. Nat. Bur. Standards Sect. B, v. 71B, 1967, pp. 111-129. MR 36 \#4841.

5. V. PEREYRA, "On improving an approximate solution of a functional equation by deferred corrections," Numer. Math., v. 8, 1966, 376-391. MR 34 \#3814. MR 28 \# 1725. 